\title{
Adrenocortical activity with repeated twice daily dosing of fluticasone propionate and budesonide given via a large volume spacer to asthmatic school children
}

\author{
B J Lipworth, D J Clark, L C McFarlane
}

\begin{abstract}
Background - In a previous single dosing study in asthmatic school children fluticasone propionate produced significantly greater suppression of overnight urinary cortisol excretion than budesonide at high doses of $800 \mu \mathrm{g} / \mathrm{day}$ or greater. The aim of this study was to assess whether conventional lower doses of both drugs cause adrenal suppression when given at steady state twice daily by large volume spacer on a microgram equivalent basis in asthmatic school children.
\end{abstract}

Methods - Eight school children of mean age $\mathbf{1 2 . 1}$ years with stable asthma of mild to moderate severity (forced expiratory volume in one second $\left(F_{E V}\right) \quad 78.6 \%$ predicted, mid forced expiratory flow rate $\left(\mathrm{FEF}_{25-75}\right) \mathbf{7 2 . 5 \%}$ predicted), on $400 \mu \mathrm{g} /$ day or less of inhaled corticosteroid, were studied in a single blind (investigator blind), placebo controlled, crossover design comparing inhaled budesonide and fluticasone propionate $100 \mu \mathrm{g}$ bid and $200 \mu \mathrm{g}$ bid. Each dose was given at 08.00 hours and 20.00 hours for four days by metered dose inhaler via their respective large volume spacers with mouth rinsing. Measurements were made of overnight urinary cortisol and creatinine excretion after the eighth dose.

Results - Neither drug produced significant suppression of overnight urinary cortisol or cortisol/creatinine excretion compared with pooled placebo and there were no differences between the drugs. Only one subject with each drug at $200 \mu \mathrm{g}$ twice daily had abnormally low urinary cortisol excretion of $<10 \mathrm{nmol} / 12$ hours. Ratios for the fold difference between active treatment versus placebo for urinary cortisol excretion were (as means and $95 \%$ confidence intervals for difference): budesonide $100 \mu \mathrm{g}$ bid 1.03 (95\% CI 0.46 to 1.61), budesonide $200 \mu \mathrm{g}$ bid 1.04 (95\% CI 0.62 to 1.46$)$; fluticasone $100 \mu \mathrm{g}$ bid 1.11 ( 0.45 to 1.77$)$, fluticasone $200 \mu \mathrm{g}$ bid 1.12 (0.78 to 1.47$)$. Likewise, there were no significant differences in overnight urinary cortisol/creatinine excretion.

Conclusions - With repeated twice daily administration at steady state across a dose range of 200-400 $\mu \mathrm{g} /$ day no evidence of significant adrenal suppression was found using the sensitive marker of overnight urinary cortisol excretion for either flu- ticasone propionate or budesonide given via a large volume spacer. These results emphasise the good safety profile in children of these inhaled steroids at conventional dose levels, which have proven antiasthmatic efficacy.

(Thorax 1997;52:686-689)

Keywords: adrenal activity, chronic dosing, inhaled corticosteroids, asthmatic children, fluticasone propionate, budesonide.

Inhaled corticosteroids have a well established role as first line preventive anti-inflammatory treatment in the treatment of childhood asthma. ${ }^{12}$ However, increased use of inhaled corticosteroids has focused greater attention on their systemic side effect profiles. Of these systemic effects, adrenal suppression is one of the most sensitive accessible markers currently available.

In a previous study in asthmatic children of similar severity we have found significant differences between inhaled fluticasone propionate and budesonide for the degree of adrenal suppression induced when they were given on a microgram equivalent basis. ${ }^{3}$ This was seen with single evening doses of $800 \mu \mathrm{g}$ or greater, with fluticasone causing significant adrenal suppression whereas budesonide caused none. These differences between single doses of fluticasone and budesonide can be explained by the pharmacological properties of fluticasone which has both greater glucocorticoid receptor potency and receptor residency time than budesonide. ${ }^{45}$

However, of greater clinical relevance is the degree of adrenal suppression seen at steady state with repeated twice daily dosing as this more accurately reflects what may be expected in everyday clinical practice. Although high doses of inhaled fluticasone propionate outwith the conventional dose range are known to cause clinically relevant growth and adrenal adverse effects, ${ }^{6}$ most asthmatic children will be maintained on doses of no more than $400 \mu \mathrm{g} /$ day. The aim of this study was therefore to investigate steady state suppressive effects with inhaled fluticasone propionate and budesonide on adrenal activity across a clinically relevant dose range of $100-200 \mu \mathrm{g}$ twice daily in asthmatic children.

\section{Methods}

PATIENTS

Eight asthmatic children (outpatients) of mean (SE) age $12.1(0.5)$ years completed the study. 
All cases were considered to have stable asthma of mild to moderate severity. Their mean forced expiratory volume in one second $\left(\mathrm{FEV}_{1}\right)$ was 1.99 (0.14) 1, 78.6 (4.7)\% of predicted, and mid forced expiratory flow rate $\left(\mathrm{FEF}_{25-75}\right)$ was $2.18(0.24) \mathrm{l} / \mathrm{s}, 72.5(7.5) \%$ of predicted. Both parents and children gave written informed consent. Approval for the study was given by the Tayside medical ethics committee for measurement of overnight urinary cortisol excretion but not plasma cortisol levels. All the children were continent of urine day and night. The subjects were taking $400 \mu \mathrm{g} /$ day or less of inhaled corticosteroid (median dose $400 \mu \mathrm{g} /$ day), beclomethasone dipropionate (Becodisk) $400 \mu \mathrm{g} /$ day (six patients) and fluticasone propionate (Diskhaler) $200 \mu \mathrm{g} /$ day (two patients). None had received oral corticosteroids within the preceding six months.

PROTOCOL

A single blind (investigator blind), placebo controlled, randomised crossover design was used with twice daily dosing over four days at each dose level. The duration of each treatment was chosen to ensure steady state drug levels. Subjects attended an initial screening visit where $\mathrm{FEV}_{1}$ and $\mathrm{FEF}_{25-75}$ were measured using a Vitalograph Compact spirometer (Vitalograph Ltd, Buckingham, UK), having withheld inhaled bronchodilators for eight hours. Detailed instruction in correct usage of metered dose inhaler (MDI) and spacer combinations were given according to the manufacturers' recommendations.

Subjects were then randomised to receive treatment for eight days in crossover fashion with either fluticasone propionate (Flixotide MDI, $50 \mu$ ger actuation, Allen and Hanburys, Uxbridge, Middlesex, UK), $100 \mu \mathrm{g}$ and $200 \mu \mathrm{g}$ twice daily or budesonide (Pulmicort MDI, $50 \mu \mathrm{g}$ per actuation, Astra Pharmaceuticals, Kings Langley, Herts, UK) $100 \mu \mathrm{g}$ and $200 \mu \mathrm{g}$ twice daily, each dose being given sequentially for four days. These were given in conjunction with their respective large volume spacer (Nebuhaler or Volumatic) according to manufacturers' instructions, using single puffs with no delay. Correct use of the spacer was checked prior to inclusion into the study as well as at each study visit. Randomisation was in balanced blocks with four given fluticasone first and four receiving budesonide first. Placebo MDI (plus spacer) was given for four days prior to the start of each of the two eight day treatment periods. There was at least a 12 day washout period between the two 12 day sequences during which the patients resumed their standard inhaled steroid therapy.

The aerosol canisters and plastic actuators were masked and placed in identical boxes. Each treatment box was dispensed by a different person from the investigator in order to keep the investigator blinded. Subjects omitted their usual steroid inhaler for the duration of each 12 day sequence. Mouth rinsing was performed after each two puffs of their study inhaler. After seven doses at each dose level, immediately before taking the eighth dose at
20.00 hours, subjects emptied their bladder and collected all their overnight urine for 12 hours until 08.00 hours the following morning. No further steroid or bronchodilator inhalers were taken until completion of the urine collection.

\section{MEASUREMENTS}

Overnight 12 hour urinary free cortisol excretion and creatinine excretion were measured. The urinary cortisol was measured using a commercial radioimmunoassay kit (Incstar, Wokingham, Berkshire, UK) with an intraassay and interassay coefficient of variability for analytical imprecision of $9.94 \%$ and $9.50 \%$, respectively. Urinary creatinine was measured on a Cobas-Bio autoanalyser (Roche Products Ltd, Welwyn Garden City, Herts, UK) and the intraassay and interassay coefficients of variability for analytical imprecision were $4.55 \%$ and $0.63 \%$, respectively. We have previously reported an intrasubject coefficient of variation of $5.4 \%$ for measurement of overnight urinary cortisol excretion in asthmatic children. ${ }^{3}$

\section{STATISTICAL ANALYSIS}

The power of the study was $80 \%$ in order to detect a $30 \%$ difference from placebo in overnight urinary cortisol excretion on the basis of a previous study with budesonide and fluticasone propionate in asthmatic children. ${ }^{3}$ All data were analysed using a Statgraphics software package (STSC Software Group, Rockville, Maryland, USA). The data were initially subjected to a plot to assess whether they conformed to a normal distribution and were then analysed by comparing the pooled placebo with both drugs at both dose levels using a multifactorial analysis of variance (MANOVA) with treatments, doses, subjects, and periods as factors. This was followed by Bonferroni multiple range testing in order to obviate multiple pairwise comparisons. The urinary cortisol/creatinine ratio data were analysed as trimmed means to account for three outlying data points above a ratio of 20 (these are depicted in fig 2 , one for budesonide $100 \mu \mathrm{g}$ bid, one for fluticasone $100 \mu \mathrm{g}$ bid, and one for fluticasone $200 \mu \mathrm{g}$ bid). The cortisol/creatinine data conform to a normal distribution when analysed in this way. A probability value of $<0.05$ (twotailed) was accepted as being significant. Ratios were calculated for the differences between each active treatment and placebo and expressed as a fold difference with $95 \%$ confidence interval (for intra-individual differences).

\section{Results}

There were no significant differences between the first and second placebos given in sequence for either overnight urinary cortisol excretion (that is, placebo 1 versus placebo 2 ): $22.5 \mathrm{nmol} / 12 \mathrm{~h}$ versus $23.5 \mathrm{nmol} / 12 \mathrm{~h}$ or for overnight urinary cortisol/creatinine excretion: $6.84 \mathrm{nmol} / \mathrm{mmol}$ versus $6.28 \mathrm{nmol} / \mathrm{mmol}$. There were also no significant differences between placebo values prior to each treatment 


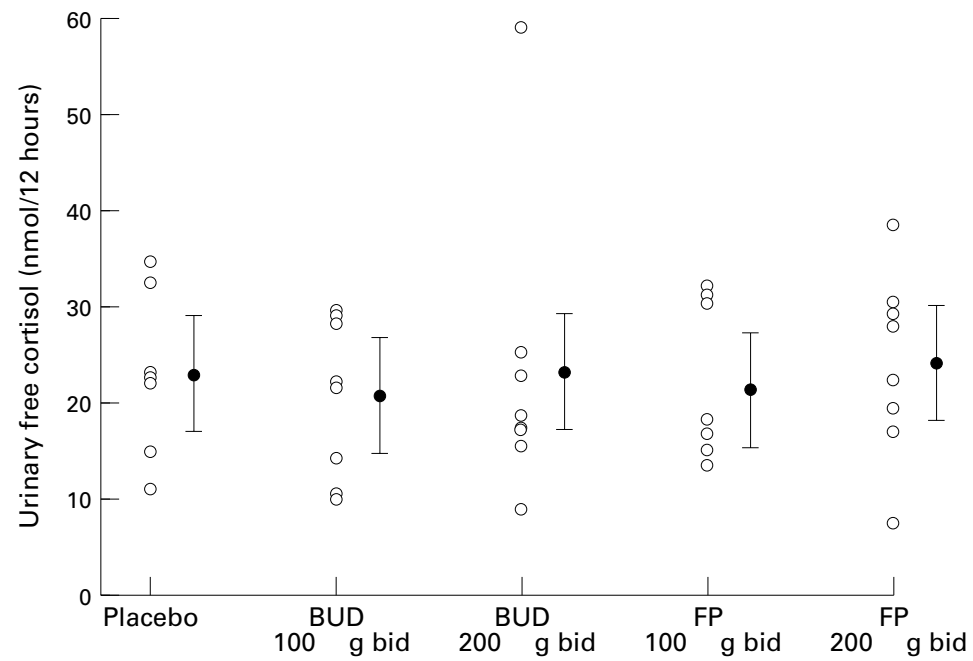

Figure 1 Individual values for overnight urinary free cortisol excretion at each dose level for repeated steady state dosing with budesonide (BUD) and fluticasone propionate (FP) or pooled placebo. No significant differences were found between the treatments or versus placebo. Values are shown as means and 95\% CI.

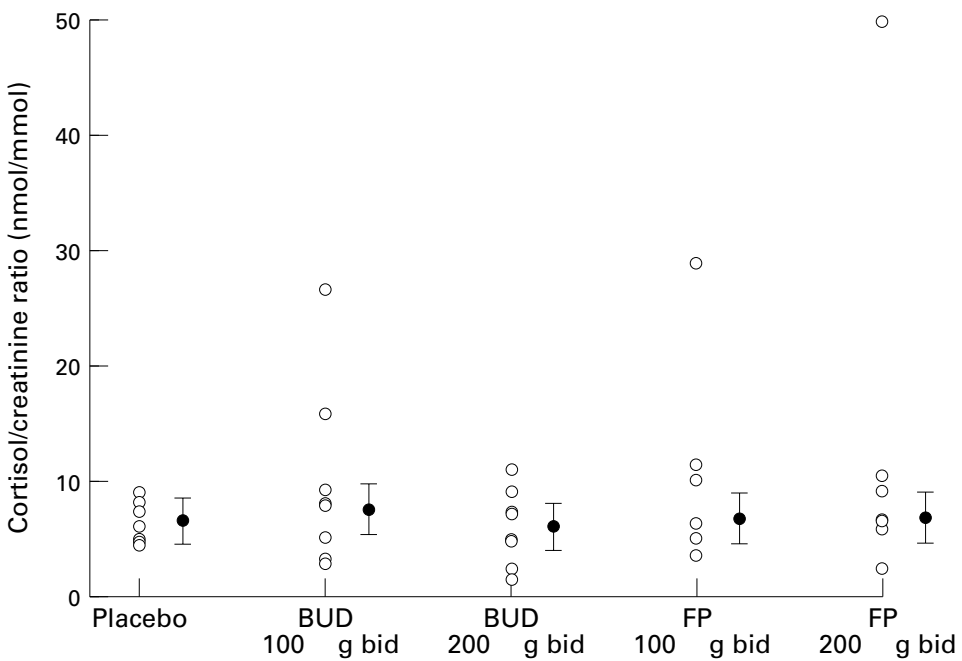

Figure 2 Individual values for overnight urinary cortisol/creatinine ratio at each dose level for repeated steady state dosing with budesonide (BUD) and fluticasone propionate (FP) or pooled placebo. No significant differences were found between the treatments or versus placebo. Values are shown as trimmed means and 95\% CI. There were three outlying data points with a ratio of $>20$ which were removed when calculating the trimmed means. compared with placebo. There were also no significant differences between the two drugs. These data are depicted in figs 1 and 2 as means and individual values to show dispersion. The ratios for suppression with active drug versus placebo are shown in table 1 as means and $95 \%$ CI for within subject differences. There were two low urinary cortisol results $(<10 \mathrm{nmol} / 12 \mathrm{~h})$, one each for fluticasone $200 \mu \mathrm{g}$ bid and budesonide $200 \mu \mathrm{g}$ bid.

\section{Discussion}

The results of this study show that inhaled budesonide and fluticasone propionate at doses up to $200 \mu \mathrm{g}$ twice daily via large volume spacer do not cause any detectable adrenal suppression at steady state using the sensitive marker of overnight urinary cortisol excretion. As a consequence of the relatively small sample size it is conceivable that we may have missed a change in overnight urinary cortisol excretion amounting to $30 \%$ or less. Nonetheless, in terms of clinical relevance only one patient with each drug at $200 \mu \mathrm{g}$ twice daily had an abnormally low overnight urinary cortisol excretion $(<10 \mathrm{nmol} / 12 \mathrm{~h})$. These findings should offer reassurance to both clinicians and parents as to the safety of inhaled corticosteroids at conventional low doses in light of recent case reports regarding the adverse effects of high doses of inhaled fluticasone in asthmatic children. ${ }^{6}$ It is worth noting that, currently in the $\mathrm{UK}$, the highest licensed recommended daily dose of budesonide in children is $800 \mu \mathrm{g} /$ day compared with $200 \mu \mathrm{g} /$ day for fluticasone propionate.

We have previously found, in asthmatic children, differences between single evening doses of inhaled fluticasone propionate and budesonide of $800 \mu \mathrm{g}$ or more in terms of adrenal suppression, with fluticasone causing detectable suppression whilst budesonide caused none even with a single dose of $1250 \mu \mathrm{g} .{ }^{3}$ These results were in keeping with those of Todd et $a l$ who also found marked adrenal suppression with high dose ( $\geq 1000 \mu \mathrm{g} /$ day) inhaled fluticasone. ${ }^{6}$ We have also found similar changes in a single evening dosing comparison of fluticasone and budesonide in adult asthmatics ${ }^{7}$ which showed a difference in plasma cortisol levels at 08.00 hours between the drugs at doses above $1000 \mu \mathrm{g}$. However, with repeated twice daily dosing in adult asthmatics differences in suppression of 08.00 plasma cortisol levels occurred with doses as low as $250 \mu \mathrm{g}$ twice daily with a mean treatment difference of $23 \%$ for cortisol suppression at 08.00 hours. $^{8}$

Similar results have also been seen in studies on healthy adult volunteers using plasma corti-

Table 1 Overnight urinary cortisol excretion and cortisol/creatinine excretion in asthmatic children given budesonide (BUD) or fluticasone propionate (FP) in doses of $100 \mu \mathrm{g}$ and $200 \mu \mathrm{g}$ twice daily

\begin{tabular}{|c|c|c|c|c|}
\hline & BUD $100 \mu \mathrm{g}$ bid & BUD $200 \mu \mathrm{g}$ bid & FP $100 \mu \mathrm{g}$ bid & FP $200 \mu \mathrm{g}$ bid \\
\hline Overnight urinary cortisol & 1.03 & 1.04 & 1.11 & 1.12 \\
\hline Overnight urinary cortisol/creatinine & $\begin{array}{l}(0.46 \text { to } 1.61) \\
(0.43 \text { to } 2.23)\end{array}$ & $\begin{array}{l}0.99 \\
(0.47 \text { to } 1.50)\end{array}$ & $\begin{array}{l}0.99 \\
(0.66 \text { to } 1.32)\end{array}$ & $\begin{array}{l}(0.18 \text { to } 1.4 t) \\
(0.72 \text { to } 1.59)\end{array}$ \\
\hline
\end{tabular}

Ratios for fold difference between each active treatment versus placebo are shown as means (95\% CI). Those $95 \%$ CI which include unity indicate no significant difference between active treatment and placebo. 
sol $\mathrm{AUC}_{0-20}$, revealing an accentuation of the differences between fluticasone and budesonide with repeated dosing compared with single doses. ${ }^{9}$ Boorsma et al also found differences between the two drugs in healthy volunteers with a $20 \%$ treatment difference in suppression of plasma cortisol $\mathrm{AUC}_{0-24}$ at a dose of $200 \mu \mathrm{g}$ twice daily. ${ }^{10}$ These results are explained by the pharmacological properties of these drugs as, with chronic dosing, the longer plasma elimination half life of fluticasone of 14.4 hours $^{11}$ compared with 2.3 hours for budesonide ${ }^{12}$ and the greater lipophilicity of fluticasone ${ }^{13}$ will result in greater plasma and tissue retention. This, in conjunction with increased steroid potency and affinity for fluticasone, would be expected to accentuate the differences found between fluticasone and budesonide from the level of suppression already seen with single dosing.

The absence of detectable adrenal suppression with chronic dosing in children therefore appears initially to be somewhat surprising in view of our previous paediatric single dosing results and the marked step up seen between single and chronic dosing in adults. However, one possible explanation for this is the increased plasma clearance of corticosteroids in children compared with adults which has been demonstrated with budesonide. Pedersen et al found that plasma clearance of budesonide was $40 \%$ greater in children with a shorter elimination half life of 1.5 hours compared with 2.3 hours in adults. ${ }^{14}$ It is conceivable, although we are unaware of any confirmatory data, that the steady state clearance of fluticasone also differs between children and adults. Another major factor is the marked difference in dosage between our paediatric single and repeated dosing designs. The dose of fluticasone which showed a difference in adrenal suppression compared with budesonide as single evening doses of these drugs was $800 \mu \mathrm{g}$ whereas the highest evening dose in the present chronic dosing study was only $200 \mu \mathrm{g}$.

There are few peer reviewed published data on the systemic activity of fluticasone propionate compared with other inhaled corticosteroids in children. Our results are in line with the findings of Wolthers et al who studied the effects of chronic dosing with budesonide in doses of $200-800 \mu \mathrm{g} /$ day via an MDI plus Nebuhaler and found no evidence of a dose response effect on 24 hour urinary free cortisol excretion. ${ }^{15}$ Fluticasone has also been assessed with chronic dosing at $100 \mu \mathrm{g}$ twice daily via an MDI and spacer and no changes were seen in plasma cortisol levels at $08.00-10.00$ hours. ${ }^{16}$ In an abstracted comparison of fluticasone and budesonide given as dry powder inhalers in doses of $200-400 \mu \mathrm{g} /$ day no significant effects were found on a battery of bone, growth, and collagen markers. ${ }^{17}$ In contrast, beclomethasone dipropionate in doses of $400 \mu \mathrm{g} /$ day and $800 \mu \mathrm{g} /$ day caused significantly lower short term growth velocity (knemometry) than fluticasone in a dose of $200 \mu \mathrm{g} /$ day given by dry powder inhaler. ${ }^{18}$

Finally, it is also important to consider the amount of fine particle dose delivered from the device as this will determine the lung bio- availability and hence systemic activity. ${ }^{19}$ In this respect the addition of a Nebuhaler to the MDI for budesonide has been shown to increase the in vitro respirable fraction from $8 \%$ to $27 \%$ using an Andersen sampler in conjunction with an anatomical child throat. ${ }^{20}$ Furthermore, in vitro data using identical methodology have shown that the respirable fraction for budesonide Turbohaler is $30 \%{ }^{21}$ so it is likely that the data for budesonide via a Nebuhaler $(27 \%$ respirable fraction) can be extrapolated to the Turbohaler.

In conclusion, we have found that twice repeated daily dosing with inhaled budesonide and fluticasone propionate given via a large volume spacer in a dose range of $200-400 \mu \mathrm{g}$ day did not cause any detectable adrenal suppression in asthmatic children.

This study was funded by a grant from the Astra Foundation (UK).

1 British Thoracic Society. Chronic asthma in adults and children. Thorax 1993;48(Suppl):S1-24.

2 Barnes PJ. Inhaled glucocorticoids for asthma $N$ Engl $\mathscr{F}$ Med 1995;332:868-75.

3 Clark DJ, Clark RA, Lipworth BJ. Adrenal suppression with inhaled budesonide and fluticasone propionate given by large volume spacer to asthmatic children. Thorax 1996; 51:941-3.

4 English AF, Neate MS, Quint DJ, Sareen M. Biologica activities of some corticosteroids used in asthma (abstract). Am f Respir Crit Care Med 1994;149(Suppl):A212.

5 Hogger P, Rohdewald P. Binding kinetics of fluticasone propionate to the human glucocorticoid receptor. Steroids 1994;59:597-602

6 Todd G, Dunlop K, McNaboe J, Ryan MF, Carson D, Shields MD. Growth and adrenal suppression in asthmatic children treated with high-dose fluticasone propionate. Lancet 1996;348:27-9.

7 Clark DJ, Grove A, Cargill RI, Lipworth BJ. Comparative adrenal suppression with inhaled budesonide and fluticasone propionate in adult asthmatic patients. Thorax 1996;51:262-6.

8 Clark DJ, Lipworth BJ. Adrenal suppression with chronic dosing of fluticasone propionate compared with budesonide in adult asthmatic patients. Thorax 1997;52:55-8.

9 Lonnebo A, Grahnen A, Jansson B, Brundin RM, Ling Andersson A, Eckernas SA. An assessment of the systemic effects of single and repeated doses of inhaled fluticasone propionate and inhaled budesonide in normal volunteers. Eur f Clin Pharmacol 1996;49:459-63.

10 Boorsma M, Andersson N, Larsson P, Ullman A. Assessment of the relative systemic potency of inhaled fluticasone and budesonide. Eur Respir f 1996;9:1427-32.

11 Thorsson L. Dahlstrom K, Edsbacker S, Kallen A, Paulson J, Wiren JE. Pharmacokinetics and systemic effects of inhaled fluticasone propionate in healthy subjects. $B r \mathscr{F}$ Clin Pharmacol 1997;43:155-61.

12 Thorrson L, Edsbacker S, Conradson TB. Lung deposition of budesonide from Turbuhaler is twice that from presof budesonide from Turbuhaler is twice that from pres-
surised metered-dose inhaler. Eur Respir f 1994;7:183944 .

13 Wurthwein G, Rehder S, Rohdewald P. Lipophilicity and receptor affinity of glucocorticoids. Pharm Ztg Wiss 1992 4:161-7.

14 Pedersen S, Steffensen G, Ekman I, Tonnesson M, Borga A. Pharmacokinetics of budesonide in children with asthma. Eur f Clin Pharmacol 1987;31:579-82.

15 Wolthers OD, Pedersen S. Measures of systemic activity of inhaled glucocorticoids in children: a comparison of urine cortisol excretion and knemometry. Respir Med 1995;89: $347-9$.

16 Gustafsson P, Tsanakas J, Gold M, Primhak R, Radford M Gillies E. Comparison of the efficacy and safety of inhaled fluticasone propionate $200 \mu \mathrm{g} /$ day with inhaled beclomethasone dipropionate $400 \mu \mathrm{g} / \mathrm{day}$ in mild and moderate asthma. Arch Dis Child 1993;69:206-11.

17 Agertoft L, Pedersen S. Bone, growth and collagen markers in children treated with fluticasone propionate and budesonide (abstract). Eur Respir f 1996;9(Suppl 23): 295s.

18 Wolthers O, Pedersen S. Short term growth during treatment with inhaled fluticasone propionate and beclomethasone with inhaled fluticasone propionate and becl
dipropionate. Arch Dis Child 1993;68:673-6.

19 Lipworth BJ. Airway and systemic effects of inhaled corticosteroids in asthma: dose-response relationship. Pulm Pharmacol 1996;9:19-27.

20 Berg E. In vitro properties of pressurised metered dose inhalers with and without spacer devices. 7 Aerosol Med 1995;8(Suppl 3):S3-11

21 Olsson B. Aerosol particle generation from dry powder inhalers: can they equal pressurised metered dose inhalers? $\mathcal{f}$ Aerosol Med 1995;8(Suppl 3):S13-18. 